APS/123-QED

\title{
Understanding the electroluminescence emitted by single molecules in scanning tunneling microscopy experiments
}

\author{
John Buker and George Kirczenow \\ Physics Department, Simon Fraser University, \\ Burnaby, British Columbia, Canada V5A 1 S6
}

(Dated: October 26, 2018)

\begin{abstract}
We explore theoretically the electroluminescence of single molecules. We adopt a local-electrode framework that is appropriate for scanning tunneling microscopy (STM) experiments where electroluminescence originates from individual molecules of moderate size on complex substrates: Couplings between the STM tip and molecule and between the molecule and multiple substrate sites are treated on the same footing, as local electrodes contacting the molecule. Electron flow is modelled with the Lippmann-Schwinger Green function scattering technique. The evolution of the electronic energy levels of the molecule under bias is modelled assuming the total charge of the molecule to be invariant, consistent with Coulomb blockade considerations, but that the electronic occupations of the molecular HOMO and LUMO levels vary with changing bias. The photon emission rate is calculated using Fermi's golden rule. We apply this theoretical approach to the STM/Znetioporphyrin $/ \mathrm{Al}_{2} \mathrm{O}_{3} / \mathrm{NiAl}(110)$ system, and simulate various configurations of coupling strength between the molecule and substrate. We compare our results to the experimental observations of Qiu, Nazin and Ho [Science 299, 542 (2003)] for this system and find that our model provides a comprehensive explanation of a multitude of previously unexplained observations. These include the different types of current-voltage characteristics (CVC's) that are observed experimentally, the observed association of electroluminescence with some CVC's and not others and key properties of the observed photon spectra. Theoretical predictions are presented for further single-molecule electroluminescence experiments.
\end{abstract}

PACS numbers: Valid PACS appear here 


\section{INTRODUCTION}

In the past 15 years, molecular electronics has become a field of intense interest for fundamental research, with potential applications in the creation of nanoscopic devices [1, 2, 3]. At the same time, great progress has been made in the creation of nanoscale photonic devices such as those based on photonic band gap materials[4].

The scanning tunneling microscope (STM) is proving immensely useful in bridging the gap between these two fields. In STM experiments on clean surfaces, light emission has frequently been observed: Systems with an STM tip over a metallic [5, 6] or semiconducting [7, 8] surface are known to give off light due to the decay of plasmons. Enhanced photon emission has been observed when molecules are placed inside the tip-substrate junction[9, 10, 11, 12, 13, 14]. However, it was unclear until recently whether the stronger emission was limited to an amplification of the plasmon-based emission seen on metallic surfaces [15, 16], or whether there could in some cases be a different, inherently molecular emission mechanism at work.

Recently, it has been definitively demonstrated through STM experiments that electric current flow through a molecule may indeed cause the molecule to luminesce[17, 18] due to molecular orbital electronic transitions. This phenomenon, bridging the areas of photonics and molecular electronics, is a promising step towards an emerging field of single-molecule optoelectronics.

Much insight into the electronic properties of these STM/molecule/substrate systems has been obtained by directly studying electric current, for example through the comparison of experimental and theoretical STM topographs and current-voltage (I-V) curves. In recent years, with photonic properties of STM/molecule/substrate systems also being studied, a photon detector and spectrometer have been added to the standard STM apparatus. Simultaneous photon emission and electric current measurements have the potential to greatly enhance our understanding of these systems. A theoretical understanding of single molecule electroluminescence, however, is still in the earliest stages [19, 20, 21] and contact between theory and any specific experiment has not been made. The purpose of this article is to begin to bridge this divide between theory and single-molecule electroluminescence experiments.

The basic idea of molecular electroluminescence as observed in STM experiments is as follows: By positioning an STM tip above a single molecule on a substrate, and applying a bias voltage between the tip and substrate, electron transmission through the molecule 
may occur, mediated by the molecule's electronic orbitals, and the molecule may be found to luminesce. In a simplified picture, when a bias voltage is applied, the molecule moves out of equilibrium, with a flux of electrons passing through it. If two molecular orbitals are located in the energy window between the electrochemical potentials of the STM tip and substrate, they will both be partially occupied and, if optical transitions between them are not forbidden, transitions from the higher-energy orbital to the lower-energy orbital will occur, resulting in photon emission[19].

It has been predicted [19] and confirmed experimentally [18] that the relative coupling strengths of the molecule to the electron source and drain greatly affect molecular electroluminescence. If the coupling strengths are highly asymmetric, photon emission is severely quenched. [19] Thus, in STM/molecule/substrate experiments where the STM-molecule coupling is normally weak, a thin insulating 'spacer' layer between the molecule and metallic substrate can enhance photon emission by reducing the strength of the molecule-substrate coupling and making it comparable with the molecule-STM coupling. Conversely, this spacer layer has also been shown to strongly suppress plasmon-mediated photon emission, and thus facilitate resolving molecular electroluminescence from the background plasmon-mediated photon emission that may be present even in the absence of a molecule between the STM tip and substrate. For instance, Qiu, Nazin and Ho have studied electroluminescence of $\mathrm{Zn}(\mathrm{II})$-etioporphyrin I using a $5 \AA$-thick aluminum oxide insulating layer below the molecule (STM/Zn-etioporphyrin $/ \mathrm{Al}_{2} \mathrm{O}_{3} / \mathrm{NiAl}(110)$ )[17]. In these experiments, the STM image, the measured I-V curve and the observation of molecular electroluminescence all depend on the precise location of the molecule on the $\mathrm{Al}_{2} \mathrm{O}_{3} / \mathrm{NiAl}(110)$ substrate.

In order to theoretically model systems such as these, where there is a thin insulating 'spacer' layer[27] that has a complex atomic structure and a local geometry under the molecule that is not measured experimentally but transmits electrons nonuniformly, a local electrode approach has proved useful [22]. By considering the tip-molecule and moleculesubstrate couplings on equal footings, as local electrodes coupled to the molecule, it has been shown that the experimentally observed location-dependent STM images of the molecules can be explained in terms of different locations of dominant molecule-substrate coupling [22]. For the STM/Zn-etioporphyrin $/ \mathrm{Al}_{2} \mathrm{O}_{3} / \mathrm{NiAl}(110)$ system, there is evidence that the out-ofplane ethyl groups of the molecule may be the locations of dominant molecule-substrate coupling, and that the strength of the coupling between each ethyl group of the molecule 
and the substrate depends on the location of these groups on the substrate [22]. Thus it differs from molecule to molecule adsorbed on the substrate. However, to date there has been no theory of electroluminescence from this system.

In this article, we extend the above local-electrode theoretical framework to the study of electroluminescence and I-V characteristics observed in the experimental system of STM/Zn-etioporphyrin/ $\mathrm{Al}_{2} \mathrm{O}_{3} / \mathrm{NiAl}(110)$.[17] We consider one local STM tip probe above the molecule, and four local substrate contacts positioned below the four ethyl groups of the molecule. By varying the coupling strengths between the molecule and each of the electrodes, differing configurations can be simulated. In this model, each electrode is represented using a one-dimensional tight-binding model, and electron flow is modelled using the Lippmann-Schwinger Green-function scattering technique. Fermi's Golden Rule is used to calculate photon emission spectra.

At the present time, there is no satisfactory first principles theory of the electronic structure of molecules that are weakly coupled to the electrodes under applied bias [23], the situation under consideration here: The ab initio approach to electrical conduction based on standard time-independent density functional theory breaks down for such systems, yielding unphysical behavior for the molecular energy levels and the transmission resonances associated with them as the applied bias is varied, and therefore incorrect calculated currentvoltage characteristics for the molecule [23]. Thus, we adopt a different theoretical approach: We use semi-empirical extended Hückel parameters [24, 25] to calculate the molecular orbitals and their energies at zero applied bias. The dependence of the molecular energy levels on the applied bias is then calculated by a self-consistent procedure based on the assumption that the net charge on the molecule does not change significantly as the bias applied between the STM tip and substrate is varied in the range of bias voltages being considered. This assumption is known to be appropriate for molecules weakly coupled to the electrodes, for example, in the Coulomb blockade regime that is not captured correctly by density functional theory. For the present system this methodology is remarkably successful, and we are able to attribute the prominent features of the experimental data (all of the peaks in the differential conductance vs. applied bias, the bias voltages at the onset of electroluminescence and the energies of peaks in the observed photon spectra) to the movement of the molecular LUMO and HOMO energy levels relative to the electrochemical potentials of the source and drain electrodes that follows directly from the requirement that the charge on 
the molecule is approximately independent of the applied bias.

The experimental conductance and electroluminescence data for this system is multifaceted, depending qualitatively on the location of the molecule being probed on the $\mathrm{Al}_{2} \mathrm{O}_{3}$ substrate[17] that has a complex microscopic structure.[26] In order to account for all of the data, we find that it is necessary to include the possibility of breaking of the fourfold symmetry of the Hamiltonian of the isolated molecule in the model. This is done phenomenologically in two different ways: In one of these (Approach A) it is assumed that the symmetry is broken by the interaction of the molecule with the complex substrate. In the other (Approach B) it is assumed that the symmetry is broken through the application of bias between the STM tip and substrate. We find that both approaches are generally successful but that Approach A is able to better account for one of the features of the experimental data than Approach B. At present, since only one experiment of this kind is available, it is difficult to judge whether this difference between the two approaches confers a substantial advantage to one of them over the other. The approaches do, however, offer different predictions for experiments that have not yet been carried out.

We find that photon emission is sensitive to the details of the molecule-substrate coupling, consistent with experimental data and the local-electrode interpretation of the experimental system. We also present calculated I-V characteristics for various coupling configurations and examine the relationship between the features found in the I-V characteristics and the occurrence and nature of the luminescence emitted by the molecule. Experimentally, photon emission was found to occur when there are two peaks in $\mathrm{dI} / \mathrm{dV}$. We find that for some coupling configurations, photon emission is predicted and the characteristic two-peak curve is obtained. For another configuration, only one peak in $\mathrm{dI} / \mathrm{dV}$ is obtained and photon yield is very low. This is also in good qualitative agreement with experiment. Finally, we present a case of very weak molecule-substrate coupling, that has not yet been achieved experimentally, in which relatively high quantum efficiencies are predicted for photon emission.

The organization of this article is as follows: In Section \we describe our model and our method of solution. In Section [I] we present our results, compare them with the experimental data of Qiu, Nazin and Ho [17] and offer some predictions that may be tested in future experiments. In Section IV we present a concise summary of the aspects of the experimental data that our theory has been able to explain and of the physical mechanisms that we have identified as being responsible for them. We also comment further on the significance of the 
present work for the fields of single-molecule electronics and optoelectronics.

\section{THE MODEL}

The present model is a generalization of the simpler models presented in Refs. 19 and 22. In the present model, the tip and substrate are represented by a tip electrode (probe) and substrate electrodes (contacts), each modelled as one-dimensional tight-binding chains. Unlike in Refs. 19 and 22 where the formalism only allows single substrate contacts, in the formalism presented here an arbitrary number of substrate contacts are allowed. (We consider cases of 4 substrate contacts in the Results section of this article.) The roughly planar molecule lies on the substrate and is positioned between the tip and substrate electrodes, so that it mediates electron flow between the tip and substrate. The electronic model Hamiltonian for this system can be divided into three parts, $H=H_{\text {electrodes }}+H_{\text {molecule }}+W$, where $W$ is the interaction Hamiltonian between the electrodes and the molecule. Generalizing the Hamiltonian of Ref. 22 to allow multiple substrate contacts, the Hamiltonian for the electrodes is given by

$$
\begin{aligned}
H_{\text {electrodes }} & =\sum_{n=-\infty}^{-1} \epsilon|n\rangle\langle n|+\beta(|n\rangle\langle n-1|+| n-1\rangle\langle n|) \\
& +\sum_{i=1}^{m} \sum_{n=1}^{\infty} \epsilon|n, i\rangle\langle n, i|+\beta(|n, i\rangle\langle n+1, i|+| n+1, i\rangle\langle n, i|),
\end{aligned}
$$

where $\epsilon$ are the site energies for the electrodes, $\beta$ is the hopping amplitude between nearestneighbor electrode atoms [28], and $|n\rangle$ and $|n, i\rangle$ represent orbitals at site $n$ of the tip probe and site $n$ of the $i$ th substrate contact, respectively. We take the electrochemical potentials of the tip and substrate electrodes to be $\mu_{T}=E_{F}+e V_{\text {bias }} / 2$ and $\mu_{S}=E_{F}-e V_{\text {bias }} / 2$, where $V_{\text {bias }}$ is the bias voltage applied between them and $E_{F}$ is their common Fermi level at zero bias. The Hamiltonian of the molecule may be expressed as

$$
H_{\text {molecule }}=\sum_{j} \epsilon_{j}\left|\phi_{j}\right\rangle\left\langle\phi_{j}\right|
$$

where $\epsilon_{j}$ is the energy of the $j$ th molecular orbital $\left(\left|\phi_{j}\right\rangle\right)$. Unlike in Ref. 22, molecular orbital energies are allowed to shift in response to an applied bias voltage. Our treatment of the effect of bias voltage on orbital energies is described in Sec. IID. The interaction 
Hamiltonian between the electrodes and molecule is given by

$$
W=\sum_{j}\left(W_{-1, j}|-1\rangle\left\langle\phi_{j}\left|+W_{j,-1}\right| \phi_{j}\right\rangle\langle-1|+\sum_{i=1}^{m}\left[W_{j,(1, i)}\left|\phi_{j}\right\rangle\left\langle 1, i\left|+W_{(1, i), j}\right| 1, i\right\rangle\left\langle\phi_{j}\right|\right]\right),
$$

where $W_{-1, j}, W_{j,-1}, W_{j,(1, i)}$, and $W_{(1, i), j}$ are the hopping amplitude matrix elements between the electrodes and the various molecular orbitals $\left|\phi_{j}\right\rangle$.

Electrons propagate in the form of Bloch waves through each electrode toward the molecule, and may undergo transmission or reflection when they encounter the molecule, contributing to the occupation of molecular orbitals in the process. Wave functions of electrons incoming from the tip probe are of the form

$$
|\psi\rangle=\sum_{n=-\infty}^{-1}\left(e^{i k n d}+r e^{-i k n d}\right)|n\rangle+\sum_{i=1}^{m} \sum_{n=1}^{\infty} t_{i} e^{i k n d}|n, i\rangle+\sum_{j} c_{j}\left|\phi_{j}\right\rangle
$$

where $d$ is the lattice spacing, $t_{i}$ are the transmission coefficients into the different substrate contacts, and $r$ is the reflection coefficient.

\section{A. Solving the system}

In order to calculate molecular-based photon emission and I-V characteristics, it is necessary to evaluate the molecular orbital coefficients and transmission amplitudes for incoming electrons. This may be done by solving a Lippmann-Schwinger equation for this system, in a similar fashion to Ref. 22 but generalized to multiple substrate contacts:

$$
|\psi\rangle=\left|\phi_{0}\right\rangle+G_{0}(E) W|\psi\rangle
$$

where $G_{0}(E)=\left(E-\left(H_{\text {electrodes }}+H_{\text {molecule }}\right)+i \delta\right)^{-1}$ is the Green function for the decoupled system (without $W$ ), and $\left|\phi_{0}\right\rangle$ is the eigenstate of an electron in the decoupled tip probe (or, more generally, the incoming electrode). $G_{0}(E)$ may be separated into the decoupled components: the tip and substrate electrodes, and the molecule. For each electrode,

$$
G_{0}^{\text {electrode }}=\sum_{k} \frac{\left|\phi_{0}(k)\right\rangle\left\langle\phi_{0}(k)\right|}{E-(\epsilon+2 \beta \cos (k d))}
$$

where $d$ is the lattice spacing and $\epsilon+2 \beta \cos (k d)$ is the energy of an electrode electron with wave vector $k$. G $G_{0}^{\text {electrode }}$ may also be expressed in an atomic orbital basis:

$$
G_{0}^{\text {electrode }}=\sum_{n=1}^{\infty} \sum_{m=1}^{\infty}\left(G_{0}^{\text {electrode }}\right)_{n, m}|n\rangle\langle m|,
$$


whose matrix elements $\left(G_{0}^{\text {electrode }}\right)_{n, m}$ are known analytically. [29] For the molecule,

$$
G_{0}^{M}=\sum_{j} \frac{\left|\phi_{j}\right\rangle\left\langle\phi_{j}\right|}{E-\epsilon_{j}}=\sum_{j}\left(G_{0}^{M}\right)_{j}\left|\phi_{j}\right\rangle\left\langle\phi_{j}\right| .
$$

For an electron incoming from the tip probe, this yields the following set of linear equations for the coefficients of $|\psi\rangle:[29]$

$$
\begin{aligned}
\psi_{-1} & =\left(\phi_{0}\right)_{-1}+\left(G_{0}^{\text {electrode }}\right)_{-1,-1} \sum_{j} W_{-1, j} c_{j} \\
\psi_{1, i} & =\left(G_{0}^{\text {electrode }}\right)_{1,1} \sum_{j} W_{(1, i), j} c_{j} \\
c_{j} & =\left(G_{0}^{M}\right)_{j}\left(W_{j,-1} \psi_{-1}+W_{j,(1, i)} \psi_{1, i}\right)
\end{aligned}
$$

where $\psi_{-1}=\langle-1 \mid \psi\rangle, \psi_{1, i}=\langle 1, i \mid \psi\rangle$, and $\left(\phi_{0}\right)_{-1}=\left\langle-1 \mid \phi_{0}\right\rangle$. The transmission probability for an electron incoming from the tip probe is given by $T=\sum_{i=1}^{m} \frac{v^{\prime}}{v}\left|t_{i}\right|^{2}$. [28] Tip probe electrons between $\mu_{T}$ and $\mu_{S}$ in energy contribute to the electric current through the molecule. Using the Landauer theory, [30] an expression for the current is obtained:

$$
I=\frac{2 e}{h} \int_{\mu_{S}}^{\mu_{T}} T\left(E, V_{\text {bias }}\right) d E .
$$

The dependence of $T$ on $V_{\text {bias }}$ is due to shifting molecular orbital energies (described in Sec. IID).

\section{B. Photon emission}

Photon emission from the molecule can be understood in terms of allowed electronic transitions from a molecular orbital to one with a lower energy. To calculate emission spectra, as in Ref. 19 we use the expression for the spontaneous emission rate of a system emitting photons into empty space, using Fermi's Golden Rule.[31] The emission rate is given by

$$
\frac{4 e^{2} \omega^{3}}{3 \hbar c^{3}}\left|\left\langle\psi_{f}|\mathbf{x}| \psi_{i}\right\rangle\right|^{2}
$$

where $\psi_{i}$ and $\psi_{f}$ represent initial and final states, and $\hbar \omega$ is their difference in energy. Unlike in Ref. 19, where photon emission is calculated for the idealized case of a twoorbital molecule, here we calculate photon emission for a system involving a real molecule 
with multiple molecular orbitals. In order to do this, we consider emission only from the molecule itself. The rate is therefore approximated by

$$
R\left(k_{i}, \omega\right)=\left.\frac{4 e^{2} \omega^{3}}{3 \hbar c^{3}}\left|\sum_{j, j^{\prime}}\right| c_{j^{\prime}, f}\right|^{2}\left|c_{j, i}\right|^{2}\left|\left\langle\phi_{j^{\prime}}|\mathbf{x}| \phi_{j}\right\rangle\right|^{2},
$$

where $i$ and $f$ label initial and final states. The relevant transition dipole moments $\left\langle\phi_{j^{\prime}}|\mathbf{x}| \phi_{j}\right\rangle$ are calculated by performing an extended Hückel dipole analysis of the molecular orbitals. [32] To calculate the emission rate as a function of photon energy, we generalize the procedure presented in Ref. 19. We must consider all electron states of the system, incoming from both the tip probe and each of the substrate contacts. Each electron state consists of an incoming wave, transmitted wave, reflected wave, and an amplitude on the molecule. See Fig. 1 for a schematic illustration. We assume here (and throughout the article) the positive bias case, with $\mu_{T}>\mu_{S}$. Since we assume the temperature to be $0 \mathrm{~K}$, all states with incoming waves from a given electrode are occupied up to the electrochemical potential of that electrode. For a transition to occur, $\psi_{f}$ must be an unoccupied state, and it must be lower in energy than $\psi_{i}$. Therefore, we consider transitions from occupied initial states (below $\mu_{T}$ ) that are incoming from the tip probe, to unoccupied final states within the electrochemical potential window (above $\mu_{S}$ ) that are incoming from one of the substrate contacts. After normalizing the wave functions and converting the sum over $k$ states (and spin) into an integral over energy, an expression for the photon emission spectrum (for a given bias voltage) is obtained:

$$
f(\omega)=\frac{1}{2 \pi} \sum_{\text {contacts }} \int_{\mu_{S}+\hbar \omega}^{\mu_{T}} \frac{R\left(k_{i}, \omega\right)}{-\beta \sin \left(k_{i} d\right)} d E_{i},
$$

where $E_{i}$ and $k_{i}$ are the initial energy and wave vector of an electron incoming from the tip probe, and $\omega$ is the frequency of the photon emitted.

\section{Electronic structure of Zn-etioporphyrin at zero bias}

The electronic structure of the Zn-etioporphyrin molecule was computed using the extended Hückel model.[25] Within this model, the energy of the highest occupied molecular orbital (HOMO) is $-11.5 \mathrm{eV}$, and the energy of the LUMO is $-10.0 \mathrm{eV}$. For a charge neutral molecule at equilibrium, weakly coupled to the electrodes, the Fermi level of the electrodes at zero bias is expected to be located between the molecular HOMO and LUMO levels. 
However, the precise location of the Fermi level is a difficult problem in molecular electronics, with differing theoretical approaches yielding differing results. In STM experiments on Zn-etioporphyrin, the appearance of a low-bias $\mathrm{dI} / \mathrm{dV}$ peak for some positions of the molecule above the substrate implies a Fermi level that is close to either the HOMO or LUMO. Since these are STM experiments, it is likely that the low-bias peak corresponds to an orbital entering the Fermi energy window by crossing $\mu_{T}$ (the electrochemical potential of the STM tip) rather than $\mu_{S}$, due to the weaker coupling of the orbital to the tip than to the substrate. Since these experiments were performed at positive bias (electron flow from tip to substrate), it is therefore likely that the Fermi level is close to the LUMO and not the HOMO.

The precise location of the Fermi level of the electrodes below the LUMO energy is likely to depend on the local geometry of the Zn-etioporphyrin $/ \mathrm{Al}_{2} \mathrm{O}_{3} / \mathrm{NiAl}(110)$ interface: A work function study [33] of $\mathrm{Al}_{2} \mathrm{O}_{3}$ on $\mathrm{NiAl}(110)$ has found that the formation of an ultrathin $\mathrm{Al}_{2} \mathrm{O}_{3}$ layer on $\mathrm{NiAl}(110)$ decreases the work function of the substrate by about 0.8 $\mathrm{eV}$, with a strong dependence on the oxide layer structure and thickness. It is therefore

reasonable to assume that, for the experiments by Qiu, et al.,[17] different locations on the $\mathrm{Al}_{2} \mathrm{O}_{3} / \mathrm{NiAl}(110)$ substrate have different local work functions, with differences on the order of a few tenths of an $\mathrm{eV}$. Due to these differences, variations in the common zero-bias Fermi energy of the tip and substrate (relative to the vacuum and also to the energies of the molecular orbitals) are likely to occur. Our calculations show that in most cases the overall qualitative picture is not sensitive to the precise location of the Fermi level below the LUMO energy. Therefore, for a qualitatively reasonable analysis of this system, we choose a zero-bias Fermi level of $-10.1 \mathrm{eV}$. We justify this reasoning more explicitly in Sec. IIIB(3), where we compare our results for this Fermi level with results obtained assuming a zero-bias Fermi level of $-10.3 \mathrm{eV}$.

\section{Molecular orbital energy-level dependences}

In order to realistically model photon emission and electric current as a function of bias voltage, it is necessary to consider the effects of bias voltage on molecular orbital energies. When a bias voltage is applied, an electric field is created between the tip and substrate, which may result in some charging of the molecule. If this occurs, the charging causes 
an electrostatic shift of the molecular energy levels that in turn severely limits the actual charging that takes place. [34] Generalizing the minimal charging approximation presented in Ref. 19, we phenomenologically approximate the shift of the molecular levels in response to the applied bias by adjusting $\epsilon_{j}$ for each molecular orbital so as to maintain the net charge that the molecule has at zero bias. The net electronic charge is calculated by summing over all occupied electron states (including spin) incoming from each electrode. This sum is converted into an integral, and an expression for the charge is obtained:

$$
Q=\frac{1}{2 \pi}\left(\int^{\mu_{T}} \sum_{j} \frac{\left|c_{j}\left(E, V_{\text {bias }}\right)\right|^{2}}{-\beta \sin (k d)} d E+\sum_{\text {contacts }} \int^{\mu_{S}} \sum_{j} \frac{\left|c_{j}\left(E, V_{\text {bias }}\right)\right|^{2}}{-\beta \sin (k d)} d E\right),
$$

where the molecular orbital energies $\epsilon_{j}$ (and therefore $c_{j}$ ) change with $V_{\text {bias }}$ in such a way that $Q=$ constant.

\section{Approach $A$}

One approach to treating the bias dependence of molecular orbital energies $\epsilon_{j}$ (which we will call Approach A) is to assume an equal bias dependence for the shifts in energy of each molecular orbital. This simple approach to charging yields physically reasonable behaviour

of the molecular orbital energy levels with bias. However, by itself, it is insufficient to explain many of the experimentally observed STM I-V characteristics and photon emission results for the molecule $\mathrm{Zn}$-etioporphyrin on $\mathrm{Al}_{2} \mathrm{O}_{3} / \mathrm{NiAl}(110)$. This may be because $\mathrm{Zn}$ etioporphyrin has a two-fold degenerate lowest unoccupied molecular orbital (LUMO) that is likely to lose its degeneracy when the molecule is placed on a region of the complex surface where the molecule-substrate interaction is not fourfold-symmetric. After including such a substrate-dependent splitting in the zero-bias electronic structure of the LUMO, this approach yields interesting results that are consistent with the experimental data.

\section{Approach B}

Another approach (Approach B) is to consider the bias dependence of the different orbital energies in a slightly more complex way. Since Zn-etioporphyrin is a planar molecule and all of the relevant orbitals except for the LUMO have fourfold symmetry, the electric field from the STM tip affects each of the fourfold symmetric orbitals similarly, and we adjust their 
energies by equal amounts $\alpha$. The LUMO, however, consists of two degenerate orbitals with twofold symmetry. Depending on the position of the STM tip above the molecule, as bias voltage is applied this may result in a stronger electric field effect on the energy of one of the LUMO orbitals, and a weaker effect on the other orbital. Therefore, for cases where the tip probe is positioned above a region of the molecule with a high amplitude for one LUMO orbital and a low amplitude for the other, instead of adjusting the LUMO energies by equal amounts $\alpha$ we adjust the LUMO energies by amounts of $\gamma_{1}(>\alpha)$ and $\gamma_{2}(<\alpha)$ respectively. Within the present model, the quantities $\alpha, \gamma_{1}$ and $\gamma_{2}$ all change with $V_{\text {bias }}$ such that the total molecular charge $Q$ remains constant. These quantities depend on the electrostatic geometry of the system. Therefore, for all values of $V_{b i a s}$, the ratios $\alpha: \gamma_{1}: \gamma_{2}$ are kept the same, consistent with the linearity of electrostatics. With this phenomenological approach to charging, unlike in Approach A, we do not assume there to be any zero-bias splitting of the LUMO degeneracy.

In the remainder of this paper we will present photon emission results and current-voltage (I-V) characteristics for Zn-etioporphyrin, calculated based on the above model for both Approach A and Approach B. We will show how photon emission is sensitive to details of the molecule-substrate coupling, and explore the relationship between photon emission and $\mathrm{I}-\mathrm{V}$ curve features. In addition, we will demonstrate how our model can account for many previously unexplained features of the experimental data for this system.

\section{RESULTS}

We present results for $\mathrm{Zn}(\mathrm{II})$-etioporphyrin I, coupled to a tip probe and 4 substrate contacts that we represent for simplicity by $\mathrm{Cu} s$ orbitals. The geometrical structure of the

molecule has been calculated using density-functional theory. [35] The molecule is mainly planar and oriented approximately parallel to the substrate, but contains four out-of-plane ethyl groups.

\section{A. Strong fourfold-symmetric molecule-substrate coupling}

We first consider a case where there is strong electronic molecule-substrate coupling relative to the coupling between the molecule and the STM tip, and where the molecule-substrate 
interaction is fourfold-symmetric. By 'strong coupling' we mean that the Hamiltonian matrix elements $W_{\text {electrode, }}$ between the relevant molecular orbitals and substrate contacts are about an order of magnitude greater than between the molecular orbitals and tip probe. It has been previously shown that the out-of-plane ethyl groups of the molecule are likely locations of dominant molecule-substrate coupling. [22] Therefore, four local substrate contacts (S1-S4) are positioned below the ethyl groups of the molecule, as shown in Fig. 22, [36] For Approach A (described in Sec. IID(1)), in this case we assume there is no splitting of the LUMO degeneracy, consistent with the fourfold symmetry of the molecule-substrate coupling. The tip probe is positioned (see Fig. 2) above the molecule in a lateral region that has been shown to be part of the observed high-transmission lobe pattern for the STM tip above Zn-etioporphyrin.[17, 22] For this position of the tip probe (and any position corresponding to an experimentally observed high-transmission lobe) the tip probe has a stronger electrostatic coupling to one of the degenerate twofold symmetric LUMOs than to the other, and an intermediate coupling to all other relevant orbitals. [The difference between electrostatic and electronic coupling should be noted: Electrostatic coupling refers to the change in the electrostatic potential that an electron in a molecular orbital feels due to the applied bias voltage, whereas electronic coupling refers to the Hamiltonian matrix element $W_{\text {electrode, } j}$ between an electrode and a molecular orbital. In the rest of this article, these terms will be frequently used.] Therefore, for Approach B (discussed in Sec. IID(2)), in order to model the shift of molecular orbital energies due to electrostatic effects in a phenomenological, qualitatively reasonable way, we assume the ratio $\alpha: \gamma_{1}: \gamma_{2}$ (discussed in Sec. IID) to be 3:4:2. Here, $\gamma_{1}$ corresponds to the LUMO orbital that has stronger electrostatic coupling, and $\gamma_{2}$ to the orbital that has weaker electrostatic coupling to the tip. Results presented throughout this article are not sensitive to the precise values chosen for this ratio. [37]

\section{Approach $A$}

For this strong substrate coupling case, with Approach A, photon emission is computed to be very weak. (In Sec. IIIB,C, cases will be presented where the photon yield is more than an order of magnitude greater.) This weak emission result is consistent with the quenching of emission due to asymmetric coupling of the molecule to the tip and substrate

observed experimentally [15, 16] and predicted for the general case of current-carrying molec- 
ular wires [19]. The quenching of photon emission due to asymmetry of the electronic coupling can be understood physically as follows: Looking at Fig,1, in a highly asymmetric system where the tip-molecule coupling is much weaker than the molecule-substrate coupling, electrons incoming from the tip have relatively low amplitudes for entering the molecule, and high amplitudes for exiting into the substrate. There is therefore a low amplitude $c_{j, i}$ for an electron in its initial state to be on a molecular orbital (even if the orbital is inside the Fermi energy window and close in energy to the energy of the electron), resulting in a low photon emission rate (see Eq.14).

A further possible consideration is the molecular orbital amplitude $c_{j, f}$ of an electron in its final state. If no allowed molecular orbitals are available to receive transitions (ie. inside the Fermi energy window of the system), $c_{j, f}$ will be small for all possible final states and emission will be further quenched. As we will now show, for the strong fourfold-symmetric coupling situation we consider here, this in fact is the case.

This further quenching, as well as the calculated current-voltage (I-V) curve for this case shown in Fig. 3a, can be understood by studying how the molecular orbital energies shift with bias voltage (see Fig. 3ic): The LUMO (assumed to be degenerate in this case), becomes partially (slightly) occupied at low bias as the tip electrochemical potential $\left(\mu_{T}\right)$ approaches its energy. This causes an electrostatic shift of the molecular orbital energies (discussed in Sec. IID). The LUMO then shifts upwards in energy, following $\mu_{T}$, so that the net charge on the molecule is maintained.

The result is the approximately linear I-V curve at low bias in Fig. 3a, with electron flow being mediated by the tails of the HOMO and the LUMO. There is a slight low-bias $\mathrm{dI} / \mathrm{dV}$ feature due to $\mu_{T}$ approaching the LUMO energy. At about $1.3 \mathrm{~V}$, the slope of the $\mathrm{I}-\mathrm{V}$ curve begins to increase, resulting in a peak in $\mathrm{dI} / \mathrm{dV}$. The reason for this is as follows: The HOMO begins to become partially (slightly) unoccupied, even though it is still below the substrate electrochemical potential $\left(\mu_{S}\right)$. This is because the molecule-substrate contact couplings are strong compared to the molecule-tip coupling, so the substrate has a much stronger effect on the orbital occupations than the tip, and the high-energy tail of the HOMO begins to depopulate. HOMO electrons inside the Fermi energy window contribute to current flow into the substrate, increasing the slope of the I-V curve. The orbital energies are affected slightly, with the LUMO shifting slightly lower relative to $\mu_{T}$ (but not visibly in Fig. 3re), such that the net charge on the molecule is maintained. The slight downward 
shift of the LUMO energy further increases the slope of the I-V curve. Here, electric current is very sensitive to such a shift, due to the LUMO's energy being very close to $\mu_{T}$. At about $1.4 \mathrm{~V}$, the LUMO fully enters the Fermi energy window, in the process becoming only

slightly occupied due to the much weaker coupling of the molecule to the tip (electron source electrode) than to the substrate (drain). At this point both the HOMO and LUMO orbital energies shift downwards, in such a way that the charge on the molecule remains constant (ie. the HOMO energy follows $\mu_{S}$. The HOMO energy remains below $\mu_{S}$, resulting in quenched photon emission. The I-V curve flattens, since no orbitals are entering or approaching the energy window between tip and substrate Fermi energies.

We now compare this result with experimental results obtained by Qiu et al.[17] for the $\mathrm{STM} / \mathrm{Zn}$-etioporphyrin $/ \mathrm{Al}_{2} \mathrm{O}_{3} / \mathrm{NiAl}(110)$ system. In these experiments, depending on the location of the molecule on the substrate, the molecule either luminesced or did not, with different dI/dV curves obtained for luminescent and non-luminescent cases. See Fig. (4) for the reproduced experimental curves. Here, curve A and curve B are representative of molecules that were found to luminesce. Molecules with current-voltage curves C-F did not exhibit observable luminescence. Experimentally, molecules that did not luminesce were found to have only one $\mathrm{dI} / \mathrm{dV}$ peak, usually at around $1.4 \mathrm{~V}$. This is in good qualitative agreement with the model result presented here, using Approach A, which shows only one significant $\mathrm{dI} / \mathrm{dV}$ peak that occurs at $1.4 \mathrm{~V}$ in Fig. $3 \mathrm{k}$, and very weak photon emission, that is likely not experimentally detectable.

\section{Approach B}

For the case of strong fourfold-symmetric molecule-substrate coupling, Approach B (discussed in Sec.IID) yields I-V results shown in Fig. 3b that are qualitatively similar to those in Fig. 3a that were obtained using Approach A. Photon emission is also computed to be very weak, for the same reasons as with Approach A.

With Approach B, the LUMO with the weaker electrostatic coupling to the tip (which we will refer to as LUMO2) enters the Fermi energy window at low bias (see Fig. 3 contributes very little to the electric current (see Fig. 3b), due to the very weak LUMO2-tip probe electronic coupling. The LUMO2 remains almost completely unoccupied because of the asymmetry of the LUMO2-tip and LUMO2-substrate couplings. As $\mu_{T}$ approaches the 
energy of the more strongly electrostatically and electronically coupled LUMO (LUMO1), however, the LUMO1 becomes partially (slightly) occupied and shifts in energy, following $\mu_{T}$, so that the net charge on the molecule is maintained. The result is again an approximately linear I-V curve, with electron flow being mediated by the tails of the HOMO and the LUMO1.

At about $1.5 \mathrm{~V}$, the HOMO begins to become partially (slightly) unoccupied, similarly to Approach A, increasing the slope of the I-V curve. The LUMO1 shifts slightly lower relative to $\mu_{T}$, such that the net charge on the molecule is maintained. This further increases the slope of the I-V curve. At 1.6 V, the LUMO1 fully enters the Fermi energy window, in the process becoming only slightly occupied due to the asymmetry of the coupling. As with Approach A, the orbital energies then shift downwards, in such a way that the charge on the molecule remains constant. The HOMO energy remains below $\mu_{S}$, resulting in quenched photon emission, and the I-V curve flattens.

For this case of strong molecule-substrate coupling using Approach B, there is found to be only one significant $\mathrm{dI} / \mathrm{dV}$ peak (at $1.6 \mathrm{~V}$ ) and very weak photon emission. As for Approach A, this compares well with the experimental non-luminescent cases (see Fig. 4C-F), where one $\mathrm{dI} / \mathrm{dV}$ peak is observed (at about $1.4 \mathrm{~V}$ ).

\section{B. Localized strong coupling}

Next, we consider the case where there is strong electronic coupling between the molecule and only one of the four substrate contacts. It has been suggested[22] that this type of electrode configuration is a likely possibility for the common experimental case of Fig.2B in the article by Qiu et al.[17] Significant molecular electroluminesce was observed for this experimental case.

The electrode configuration that we consider is similar to Sec.IIIA (see Fig. 2); however, in this case the substrate contacts $S_{1}, S_{2}$ and $S_{3}$ are moderately coupled to the molecule (coupling less than an order of magnitude greater than the coupling to the tip probe), and $S_{4}$ is strongly coupled. 38] The tip probe is positioned in the same lateral region as for Sec.IIIA, and again with greater electrostatic coupling to one LUMO (LUMO1) relative to the other LUMO (LUMO2). It should also be noted that, due to the twofold symmetry of the LUMO,

the strongly coupled substrate contact is electronically strongly coupled to only one of the 
LUMOs (LUMO2, in this case) and not the other (LUMO1).

\section{Approach $A$}

With Approach A, since the molecule-substrate interaction is in this case not fourfoldsymmetric, there is a splitting in the zero-bias degeneracy of the LUMO.[39]

For this case, significant photon emission is computed to occur. Fig. [5a shows the calculated emission spectrum at high bias $\left(V_{\text {bias }}=1.94 \mathrm{~V}\right)$. The spectrum corresponds to HOMO-LUMO1 (1.94 eV peak) and HOMO-LUMO2 (1.44 eV peak) transitions. The calculated I-V curve for this case, shown in Fig. [5k, has a low-bias dI/dV peak and a high-bias $\mathrm{dI} / \mathrm{dV}$ peak.

To understand the calculated photon emission spectra and I-V curves for this case, it is necessary to pay close attention to the details of the coupling of the various molecular orbitals to the electrodes. Looking at Fig,55, at low bias the LUMO2 enters the Fermi energy window, remaining almost completely unoccupied due to the strongly asymmetric coupling of the LUMO2 to the tip and substrate. In this case, however, the LUMO2 contribution to the electric current is not negligible. Current flow mediated by the LUMO2 is not drowned out by current flow mediated through the tails of the LUMO1 or the HOMO, since in this case the electronic coupling of the substrate is strongest to the LUMO2. This creates the low-bias dI/dV peak seen in Fig. 55.

The LUMO2 energy follows $\mu_{T}$ up to $0.2 \mathrm{~V}$ (see Fig. 5b). In this case, the substrate contacts have a large influence on the occupation of the LUMO2 even though the LUMO2 is well above $\mu_{S}$, because the coupling between the substrate and LUMO2 is much stronger than between the tip and LUMO2. Thus, from $0.2 \mathrm{~V}$ to $0.6 \mathrm{~V}$ the LUMO2 tracks $\mu_{S}$ and the I-V curve (Fig. 5 $\mathrm{F}$ ) is flat. At $0.6 \mathrm{~V}$, the LUMO1 approaches $\mu_{T}$ and begins to populate. In response, the energies of the orbitals rise such that no charging takes place. The tip probe has a large influence on the occupation of the LUMO1, because the coupling between the tip/substrate and LUMO1 is not highly asymmetric. From $0.6 \mathrm{~V}$ to $1.9 \mathrm{~V}$, The LUMO1 and the tail of the HOMO are the dominant sources of rising current.

The HOMO reaches $\mu_{S}$ at $V_{\text {bias }}=1.9 \mathrm{~V}$, causing an electrostatic shift in energy of the orbitals downwards, so that the LUMO1 enters the Fermi energy window and populates significantly. The HOMO reaches $\mu_{S}$ and depopulates by an equal amount. There is a resulting 
sharp increase in current, as both the HOMO and LUMO1 mediate electron transmission from tip to substrate.

Close inspection of Fig. 5e and Fig. 35c shows that, in this case, the HOMO energy comes up to $\mu_{S}$ (within the resolution of the figure) whereas for the case of Sec. IIIA (Approach A), the HOMO energy only approaches $\mu_{S}$. Hence, the depopulation of the HOMO is much greater in this case than in Sec.IIIA. Greater depopulation of the HOMO occurs, along with greater population of the LUMO1 as it enters the Fermi energy window, due to the lack of strong coupling asymmetry between the LUMO1 and the tip/substrate electrodes in this case. In this way, the zero-bias charge is maintained. Therefore, above $1.9 \mathrm{~V}$, HOMOmediated electronic states are available to receive transitions from LUMO1 and LUMO2mediated states, resulting in photon emission. Since there is a stronger coupling asymmetry between the LUMO2 and the tip/substrate electrodes than between the LUMO1 and those electrodes, LUMO2-HOMO photon emission is weaker than LUMO1-HOMO emission (see Fig. 5a), as explained in Sec.IIIA.

The onset of photon emission in this case occurs as the HOMO becomes partially unoccupied, at about $1.9 \mathrm{~V}$. Fig. 6a shows the onset of photon emission, at the spectrum peak corresponding to HOMO-LUMO2 transitions. Notice that the peak photon energy $(\approx 1.43-$ $1.44 \mathrm{eV})$ is significantly less than the Fermi gap energy $(\approx 1.93-1.94 \mathrm{eV})$. This is because the LUMO2 is deep inside the Fermi energy window at the onset voltage (see Fig. 5r). The calculated photon emission peak due to HOMO-LUMO1 transitions has the same onset voltage as the HOMO-LUMO2 emission peak. For this transition, however, photon energy is peaked close to the Fermi gap energy $(1.9 \mathrm{eV})$ because the LUMO1 and HOMO have energies close to $\mu_{T}$ and $\mu_{S}$ respectively at the onset voltage.

Comparing results for this luminescent case to experiment, the similarities are striking. Experimentally, molecules that luminesced commonly had a small $\mathrm{dI} / \mathrm{dV}$ peak at $0.2 \mathrm{~V}$ and a larger peak at around $2.0 \mathrm{~V}$ (see Fig. 4A,B). This is in excellent qualitative agreement with Fig.5c, where we see a small $\mathrm{dI} / \mathrm{dV}$ peak at $0.2 \mathrm{~V}$ and a larger peak at about $1.9 \mathrm{~V}$. Furthermore, experimental results[17] (reproduced here in Fig. 7) show the onset of photon emission occurring most commonly at about $2.2 \mathrm{~V}$, but with a photon energy peak in the spectrum about $0.5 \mathrm{eV}$ below the corresponding Fermi gap energy of $2.2 \mathrm{eV}$. This is in good agreement with Fig. 6, where at onset we find an emission peak (corresponding to the HOMO-LUMO2 transition) significantly below the Fermi gap energy. Also, comparing 
Fig. [6 with emission onset spectra for the most common experimental case (Fig. 17) we see very similar behaviour of the emission spectra tails: The high-energy tail has a sharp cutoff, while the low-energy tail does not. As bias voltage increases, the high-energy cutoff shifts upwards in energy by a similar amount. In our model, we also see this behaviour, because the Fermi energy of the substrate provides a sharp energy cutoff below which there are no available final states for a transition. This cutoff reduces the extent of the high-energy tails. There is no such cutoff reducing the extent of the low-energy tails.

Notice also that, experimentally, there is a shift in the position of the high-bias $\mathrm{dI} / \mathrm{dV}$ peak, depending on whether photon emission is observed: In Fig. 4 a peak is observed at 1.4 $\mathrm{V}$ for non-luminescent cases (C-F), and around $2.0 \mathrm{~V}$ for luminescent cases (A,B). We see the same sort of bias peak shift theoretically with Approach A: $1.4 \mathrm{~V}$ for Sec.IIIA (weak emission case) and $1.9 \mathrm{~V}$ for Sec.IIIB (strong emission case). In this way, Fig. $3 \mathrm{a}$ is similar to Fig. $4 \mathrm{C}-\mathrm{F}$, while Fig. 5 r is similar to Fig. 4A,B.

Our model further predicts a stronger HOMO-LUMO1 emission peak (the $1.94 \mathrm{eV}$ peak in Fig. 河) with the same onset voltage as the experimentally observed HOMO-LUMO2 emission peak, but with a higher peak photon energy, close to the Fermi gap energy $=e V_{\text {bias }}$. The experimental photon spectra in Ref. 17 do not extend to the photon energy energy range in which this emission peak is predicted to occur $(2.2 \mathrm{eV}$ photon energy for the experimental onset voltage of $2.2 \mathrm{~V}$ ). An experimental study testing this prediction would be very desirable.

\section{Approach B}

With Approach B, as with Approach A, significant photon emission is computed to occur in this case. Fig. 5 b shows the emission spectrum at high bias $\left(V_{\text {bias }}=1.94 \mathrm{~V}\right)$. The spectrum corresponds to HOMO-LUMO1 (1.57 eV peak) and HOMO-LUMO2 (1.30 eV peak) transitions. The I-V curve for this case, shown in Fig. 5 $\mathrm{d}$, has a low-bias dI/dV peak and a high-bias $\mathrm{dI} / \mathrm{dV}$ peak.

Looking at Fig 5 f, at low bias the LUMO2 enters the Fermi energy window. It remains almost completely unoccupied due to the strongly asymmetric coupling of the LUMO2 to the tip and substrate, but as with Approach A it still contributes to the electric current. This results in the low-bias dI/dV peak seen in Fig. $5 \mathrm{~d}$ d. At $0.2 \mathrm{~V}$ the energy of the LUMO1 
reaches $\mu_{T}$. This causes an electrostatic shift in the energy levels upwards, as shown in Fig. 5f. From $0.2 \mathrm{~V}$ to $1.6 \mathrm{~V}$, the LUMO1 and the tail of the HOMO are the dominant sources of rising current. The HOMO reaches $\mu_{S}$ at $1.6 \mathrm{~V}$, causing an electrostatic shift in energy of the orbitals downwards, so that the LUMO1 enters the Fermi energy window and populates significantly. Similarly to Approach A, the HOMO reaches $\mu_{S}$ and depopulates by an equal amount, resulting in a sharp increase in current.

For the same reasons as were explained for Approach A, for Approach B at 1.6 V HOMOmediated electronic states are available to receive transitions from LUMO1 and LUMO2mediated states, resulting in photon emission. As with Approach A, LUMO2-HOMO photon emission is weaker than LUMO1-HOMO emission (see Fig. 5b). Fig. 6b shows the onset of photon emission, around $V_{\text {bias }}=1.6 \mathrm{~V}$, at the spectrum peak corresponding to HOMOLUMO2 transitions. As with Approach A, the photon peak energy is significantly less than the Fermi gap energy.

Qualitatively, I-V and photon emission results for Approach B are similar to results for Approach A, and compare similarly well to experiment. There is one exception: With Approach $B$, there is no shift in the position of the high-bias $\mathrm{dI} / \mathrm{dV}$ peak depending on whether or not photon emission is observed: A peak is predicted at $1.6 \mathrm{~V}$ for both luminescent and non-luminescent cases, due to the very similar molecular orbital energetics for luminescent (Fig. 5f) and non-luminescent (Fig. 3d) cases. Experimentally, there is a shift in the position of the $\mathrm{dI} / \mathrm{dV}$ peak: around $1.4 \mathrm{~V}$ for the non-luminescent case and $2.0 \mathrm{~V}$ for the luminescent case (see Fig. 4)). A similar shift is found theoretically with Approach A, due to the fact that the HOMO-LUMO1 energy difference in the luminescent case (Fig. 5e) is greater than the HOMO-LUMO energy difference in the non-luminescent case (Fig. 3r).

The physical reason for this difference between Approach A and Approach B is that in Approach A the molecule-substrate coupling splits the LUMO degeneracy in the luminescent case but not in the non-luminescent case and this difference in electronic structure results in the different bias voltages at which the high bias peak in $\mathrm{dI} / \mathrm{dV}$ occurs. By contrast, in Approach B the LUMO degeneracy is lifted in both the luminescent and non-luminescent cases, so that the electronic structure of the molecule and the bias voltage at which the high bias peak in $\mathrm{dI} / \mathrm{dV}$ occurs is similar in the two cases. 


\section{Dependence on the zero-bias Fermi level}

Experimentally, different $\mathrm{dI} / \mathrm{dV}$ curves are observed depending on the location of the molecule on the substrate (see Fig. 4) [17]. Even among those molecules that luminesced ( $\mathrm{A}$ and $\mathrm{B}$ ), there are differences in $\mathrm{dI} / \mathrm{dV}$. It should be noted that, in our article, we have chosen a zero-bias Fermi level of $-10.1 \mathrm{eV}$, and that variations in the Fermi level relative to the molecular levels at zero bias are likely, depending on the location of the molecule on the surface, due to local work function variations (discussed in Sec. IIC). The dashed line in Fig. 5d shows an I-V curve (using Approach B) for an alternate zero-bias value of $E_{F}$ : $-10.3 \mathrm{eV}$ instead of $-10.1 \mathrm{eV}$. Here, the low-bias $\mathrm{dI} / \mathrm{dV}$ peak is at $0.5 \mathrm{eV}$, corresponding more closely to Fig. $4 \mathrm{~A}$ than Fig. $4 \mathrm{~B}$. It is possible that the experimental differences in low-bias $\mathrm{dI} / \mathrm{dV}$ peak locations in Qiu's Fig.2A and Fig.2B are due to different zero-bias Fermi levels caused by local work function variations on the surface. Other than the change in the lowbias $\mathrm{dI} / \mathrm{dV}$ peak location, small changes in the Fermi level yield qualitatively similar I-V and photon emission results. Therefore, in the rest of this article, we have assumed a Fermi level of $-10.1 \mathrm{eV}$.

\section{Weak fourfold-symmetric molecule-substrate coupling}

The final case we consider is weak molecule-substrate coupling, where the moleculesubstrate interaction is fourfold-symmetric, along with stronger tip-molecule coupling than in the previous cases. In this case, the electronic molecule-substrate coupling is of the same order of magnitude as the tip-molecule coupling. [40] This situation may be achieved experimentally by increasing the thickness of the oxide layer between the molecule and metal substrate by a modest amount, or by decreasing the tip-molecule distance. In our model, we both increase the molecule-substrate distance and decrease the tip-molecule distance.

\section{Approach $A$}

As in Sec.IIIA(1), we assume there is no splitting of the LUMO degeneracy. For this case, much more efficient photon emission is predicted to occur, with a photon yield two orders of magnitude higher than for Sec. IIIB. Fig. 8 a shows the emission spectrum at high bias $\left(V_{\text {bias }}=1.95 \mathrm{~V}\right)$. The peak in the spectrum corresponds to the HOMO-LUMO transition. 
Fig. 8c shows the $\mathrm{I}-\mathrm{V}$ curve for this case. There is a high-bias $\mathrm{dI} / \mathrm{dV}$ peak (at $1.45 \mathrm{~V}$ ) and no low-bias peak.

Looking at Fig. 8e, the molecular orbital energetics are similar to those for Sec.IIIA(1) (shown in Fig. 3. ). Since no orbitals enter the Fermi energy window at low bias, there is no low-bias $\mathrm{dI} / \mathrm{dV}$ peak. In this case, the $\mathrm{I}-\mathrm{V}$ curve is quite flat up to about $1.4 \mathrm{~V}$. At 1.4 V, the LUMO fully enters the Fermi energy window, becoming partially occupied. The HOMO depopulates by an equal amount, and the orbitals electrostatically shift downwards in energy with $\mu_{S}$.

Since the tip has a much stronger effect on the LUMO occupation in this case than in Sec.IIIA,B, the degree of partial population of the LUMO, and partial depopulation of the HOMO, is much greater. This results in much greater quantum efficiency for photon emission. Unlike in Sec.IIIB, the initial onset voltage for photon emission due to HOMOLUMO transitions matches the HOMO-LUMO emission peak energy.

\section{Approach $B$}

As with Approach A, with Approach B very strong photon emission is predicted to occur. Fig. 8b shows the emission spectrum at high bias $\left(V_{\text {bias }}=1.80 \mathrm{~V}\right)$. Unlike for Approach A, here there are two peaks in the spectrum, corresponding to HOMO-LUMO1 and HOMOLUMO2 transitions. Fig. 8d shows the I-V curve for this case. There are two high-bias $\mathrm{dI} / \mathrm{dV}$ peaks (at $1.2 \mathrm{~V}$ and $1.6 \mathrm{~V}$ ) and no low-bias peak.

These results can be understood by studying the behaviour of the molecular orbitals with applied bias voltage (see Fig. 8f). At low bias, the Fermi energy window approaches the LUMO1 and LUMO2. Unlike the other cases (Sec.IIIA,B), in this case the LUMO2 coupling to tip and substrate is not strongly asymmetric, and electron states from the tip have a significant effect on the charge of the orbital; therefore, the LUMO2 electrostatically shifts in energy with $\mu_{T}$ so that the zero-bias charge on the molecule is maintained. Since no orbitals enter the Fermi energy window at low bias, there is no low-bias dI/dV peak. At $1.2 \mathrm{~V}$, the HOMO energy reaches $\mu_{S}$, and the HOMO begins to depopulate. This causes an electrostatic shift in orbital energy downwards, and the LUMO2 enters the Fermi energy window, creating a $\mathrm{dI} / \mathrm{dV}$ peak at $1.2 \mathrm{~V}$. At $1.6 \mathrm{~V}$, the LUMO1 enters the Fermi energy

window, resulting in another $\mathrm{dI} / \mathrm{dV}$ peak. (This increase in current is greater than the 
increase at $1.2 \mathrm{~V}$, because the LUMO1 has stronger electronic coupling than the LUMO2 to the tip probe.) The HOMO depopulates significantly further, with the LUMO1 populating by an equal amount. (The resulting electrostatic deviation in orbital energies is too small to be visible in Fig. $8 \mathrm{f}$ because the occupation of the HOMO is very sensitive to any deviation in energy away from $\mu_{S}$.)

As with Approach A, the result is higher quantum efficiency for photon emission. Unlike in $\operatorname{Sec} \operatorname{IIIB}(2)$, the initial onset voltage for photon emission due to HOMO-LUMO2 transitions corresponds to the HOMO-LUMO2 emission peak energy. The HOMO-LUMO2 emission peak increases further once the onset voltage corresponding to the HOMO-LUMO1 emission peak is reached (due to the further depopulation of the HOMO).

A signature of this relatively efficient photon emission regime, found with both Approach $\mathrm{A}$ and Approach $\mathrm{B}$, is the lack of a low-bias $\mathrm{dI} / \mathrm{dV}$ peak. This regime has yet to be realized in STM experiments; however, it is predicted that greatly enhanced quantum efficiency could be achieved by further weakening the coupling of the molecule to the metallic substrate, or by bringing the STM tip closer to the molecule. While in our model we both increase the molecule-substrate distance and decrease the tip-molecule distance, it may be more experimentally feasible to increase the thickness of the oxide layer without bringing the tip closer to the molecule. This would cause a reduced current through the molecule. For such an experimental situation, the relevant luminescence observation is not the absolute photon emission intensity, but the quantum efficiency, or photon yield (the number of photons given off per electron passing through the molecule). This is predicted to be greatly enhanced.

\section{Discussion of Results}

Both Approach A and Approach B yield results consistent with experiment. For the case where the molecule is strongly coupled to the substrate, very weak photon emission, along with only a single high-bias $\mathrm{dI} / \mathrm{dV}$ peak, is found with both approaches. Experimentally, all molecules that did not luminesce had a single high-bias dI/dV peak signature and no low bias $\mathrm{dI} / \mathrm{dV}$ peak.

For the case where only a localized region of the molecule is strongly coupled to the substrate, both approaches yield much stronger photon emission than the first case. This is because, for a HOMO-LUMO transition, the relevant coupling asymmetry (between the tip- 
LUMO and the HOMO-substrate) is greatly reduced. Two emission peaks were found, the lower-energy peak being significantly lower in energy at onset than the energy corresponding to the onset voltage. As well, in this case both a low-bias and high-bias dI/dV peak are found. This is consistent with experiment: In the experimental case where both low-bias and high-bias $\mathrm{dI} / \mathrm{dV}$ peaks are observed, photon emission is also observed. Furthermore, there is additional evidence based on modelling of the molecular STM images[22] that this experimental case corresponds to a localized region of strong coupling of the molecule to the substrate.

One qualitative feature observed experimentally and found theoretically with Approach A is not found with Approach B: Experimentally, there is a shift in the position of the high-bias dI/dV peak, depending on whether or not photon emission is observed. This shift is predicted with Approach A but not with Approach B.

There is additional experimental evidence in support of Approach A in the form of an observed zero-bias splitting in the LUMO degeneracy of a similar molecule (magnesium porphine) above the same $\mathrm{Al}_{2} \mathrm{O}_{3} / \mathrm{NiAl}(110)$ substrate. [41] It should be noted that for this experiment, only MgP molecules with two-lobe STM images were chosen for detailed study, so the substrate-dependence of the zero-bias splitting is unknown.

There is, however, a possible physical justification for Approach B. When a bias voltage is applied, the STM tip will electrostatically affect different molecular orbitals differently. The extent of these different effects is unknown. A simple electrostatic calculation, treating the tip/substrate as a point charge and a mirror image charge, suggests small differences (typically on the order of 100ths of an eV) in the average potential for the LUMO1 and LUMO2 orbitals. Thus, while the assumptions for Approach B may indeed be qualitatively correct, the degree to which the orbital energies of the LUMO1 and LUMO2 behave differently with bias is unknown and may be small.

For the case of very weak molecule-substrate coupling, much higher quantum efficiencies for photon emission are predicted to occur. This regime has not yet been realized experimentally, and would be an intriguing avenue for further research. 


\section{CONCLUSIONS}

The local-electrode framework presented in this article coherently explains a multitude of experimental observations[17] not previously theoretically studied, for the STM/Znetioporphyrin $/ \mathrm{Al}_{2} \mathrm{O}_{3} / \mathrm{NiAl}(110)$ system. The following is a summary of these observations, with explanations based on our model results:

(i) The observed molecular-based photon emission is due to transitions between the molecular LUMO, whose degeneracy has been split by molecule-substrate and/or moleculeSTM tip interactions, and the molecular HOMO.

(ii) For some cases, low-bias dI/dV peaks are observed experimentally (see Fig. 4A,B). Our model explains these as being due to a splitting of the LUMO degeneracy, with the lower-energy LUMO entering the Fermi energy window at low bias (see Sec. IIIB).

(iii) For some cases, no low-bias dI/dV peak is experimentally observed (see Fig. 4C-F). We find that this occurs because the molecule is too strongly coupled to the substrate, with the LUMO either not entering the Fermi energy window at low bias (Approach A, see Sec.IIIA(1)), or entering the window but contributing negligibly to the current due to very weak coupling of the molecule to the tip compared to the substrate (Approach B, see Sec. IIIA $(2))$.

(iv) For cases with no low-bias peak, no photon emission is experimentally observed. This is due to strongly asymmetric tip/molecule and molecule/substrate couplings. In these cases, when a bias is applied, the HOMO stays almost fully occupied and the LUMO almost completely unoccupied (see Sec. IIIA $(1,2)$ ).

(v) There is an experimentally observed difference in the position of the high-bias dI/dV peak, between cases where photon emission is and is not observed (see Fig. 4). This is explained with Approach A by a breaking of the LUMO degeneracy only in the luminescent case (see Sec. IIIA(1) and Sec. IIIB(1)).

(vi) The experimental peak photon energy is about $0.5 \mathrm{eV}$ below $e V_{\text {bias }}$ at emission onset (see Fig. 7). This is due to splitting of the LUMO degeneracy, with the lower-energy LUMO being well inside the Fermi energy window as the energy of the HOMO approaches the window. See Sec. IIIB $(1,2)$. 
(vii) The high-energy photon emission spectra tails are steeper than the low-energy tails (see Fig 7). This is due to the substrate Fermi energy providing a sharp energy cutoff below which there are no available states to receive a transition (see Sec. $\operatorname{IIIB}(1,2)$ ).

(viii) There are significant differences in experimentally observed positions of $\mathrm{dI} / \mathrm{dV}$ peaks (see Fig 4) depending on the position of the molecule on the substrate. These differences are consistent with differing local zero-bias Fermi levels, due to local variations in the work function of the oxide-coated metal substrate (see Sec. IIIB(3)).

Our model predicts an additional photon emission peak to be found, having a peak energy close to the bias voltage at emission onset, for the case of molecular-based photon emission presented in Sec. IIIB. Experiments testing this prediction would be of interest.

We also predict that greatly enhanced quantum efficiency of photon emission could be achieved by further weakening the coupling of the molecule to the metallic substrate, or if possible by bringing the STM tip closer to the molecule (see Sec. IIIC). The spectrum for this greatly enhanced quantum efficiency could yield further clues to the relative merits of the two approaches studied in this article (see Sec.IID). For Approach A, one emission peak is predicted, and for Approach B, two peaks are predicted.

Studying the $\mathrm{STM} / \mathrm{Zn}$-etioporphyrin $/ \mathrm{Al}_{2} \mathrm{O}_{3} / \mathrm{NiAl}(110)$ system using the local-electrode theoretical framework presented in this paper has yielded a coherent explanation of a large body of experimental results for this system. Using this framework, we are able to gain a much greater understanding of single molecule electroluminescence. This is an important step towards the development of the emerging field of single molecule optoelectronics. We hope that this work inspires further experimental and theoretical research in this promising new field.

While the present theory relies heavily on phenomenology, it has allowed us to construct energy level diagrams of the evolution of the molecular HOMO and LUMO orbitals and of the electrochemical potentials of the electrodes as a function of applied bias that are physically reasonable and are consistent with both the experimentally observed currentvoltage characteristics and the experimental electroluminescence data. Thus the present work can also be viewed as a quantitative interpretation of the experimental data that is unique in that it satisfies more demanding experimental constraints than previous attempts to model experimental molecular electronic data that have focussed on experimental current- 
voltage characteristics alone. Therefore, as well as contributing to a better understanding of single-molecule optoelectronics the present work provides much needed benchmarks for the development of accurate first principles theories of the evolution of the electronic structure of molecular nanowires under bias that do not yet exist at this time. 


\section{Acknowledgments}

We are grateful to Brad Johnson and Ross Hill for helpful discussions. This research was supported by NSERC. G. K. is grateful for support in the form of a Fellowship from the Canadian Institute for Advanced Research. Some numerical computations presented in this work were performed on WestGrid computing resources, which are funded in part by the Canada Foundation for Innovation, Alberta Innovation and Science, BC Advanced Education, and the participating research institutions. WestGrid equipment is provided by IBM, Hewlett Packard, and SGI.

[1] A. Troisi and M. A. Ratner, Small 2, 172 (2006).

[2] N. J. Tao, Nature Nanotechnology 1, 173 (2006).

[3] G. Kirczenow, Molecular Nanowires and their Properties as Electrical Conductors in Handbook of Nanoscience and Technology edited by A. V. Narlikar and Y. Y. Fu, Oxford University Press, U.K., to be published.

[4] J.-M. Lourtioz, C. R. Physique 9, 4 (2008).

[5] J. H. Coombs, J. K. Gimzewski, B. Reihl, J. K. Sass, R. R. Schlittler, Jour. Microscopy 152, 325 (1988).

[6] R. Berndt, J. K. Gimzewski, and P. Johansson, Phys. Rev. Lett. 67, 3796 (1991).

[7] D. L. Abraham, A. Veider, C. Schönenberger, H. P. Meier, D. J. Arent, and S. F. Alvarado, Appl. Phys. Lett. 56, 1564 (1990).

[8] A. Downes and M. E. Welland, Phys. Rev. Lett. 81, 1857 (1998).

[9] I. I. Smolyaninov and M. S. Khaikin, Phys. Lett. A 149, 410 (1990).

[10] R. Berndt, R. Gaisch, J. Gimzewski, B. Reihl, R. Schlittler, W. Schneider, and M. Tschudy, Science 262, 1425 (1993).

[11] E. Flaxer, O. Sneh, and O. Cheshnovsky, Science 262, 2012 (1993).

[12] G. E. Poirier, Phys. Rev. Lett. 86, 83 (2001).

[13] F. Touhari, E. J. A. Stoffels, J. W. Gerristsen, H. V. Kempen, and P. Callant, Appl. Phys. Lett 79, 527 (2001).

[14] K. Sakamoto, K. Meguro, R. Arafune, M. Satoh, Y. Uehara, and S. Ushioda, Surf. Sci. 502- 
503, 149 (2002).

[15] W. L. Barnes, J. Mod. Opt. 45, 661 (1998).

[16] G. Hoffmann, L. Libioulle, and R. Berndt, Phys. Rev. B 65, 212107 (2002), and references therein.

[17] X. H. Qiu, G. V. Nazin, and W. Ho, Science 299, 542 (2003).

[18] Z.-C. Dong, X.-L. Guo, A. S. Trifonov, P. S. Dorozhkin, K. Miki, K. Kimura, S. Yokoyama, and S. Mashiko, Phys. Rev. Lett. 92, 86801 (2004).

[19] J. Buker and G. Kirczenow, Phys. Rev. B 66, 245306 (2002).

[20] M. Galperin and A. Nitzan, Phys. Rev. Lett 95, 206802 (2005); J. Chem. Phys. 124, 234709 (2006).

[21] U. Harbola, J. B. Maddox, and S. Mukamel, Phys. Rev. B73, 075211 (2006).

[22] J. Buker and G. Kirczenow, Phys. Rev. B 72, 205338 (2005).

[23] M. Koentopp, C. Chang, K. Burke, and R. Car, J. Phys. Condensed Matt. 20, 083203 (2008).

[24] J. H. Ammeter, H.-B. Bürgi, J. C. Thibeault, and R. Hoffman, J. Am. Chem. Soc. 100, 3686 (1978).

[25] The implementation used was that of G. A. Landrum and W. V. Glassy, YAeHMOP project, http://yaehmop.sourceforge.net

[26] G. Kresse, M. Schmid, E. Napetschnig, M. Shishkin, L. Köhler, and P. Varga, Science 308, $1440(2005)$.

[27] Some of the data in Ref. 17 involves Zn-etioporphyrin on bare NiAl(110), with no insulating 'spacer' layer. For this data, plasmon-based photon emission is significant and molecularbased photon emission is quenched. The model in the present article has been created to study systems involving an insulating 'spacer' layer. Therefore, our discussion of the data in Ref. 17 is limited to the cases with a 'spacer' layer, where plasmon-based emission was shown to be negligible experimentally,[17] and plasmon effects are not considered in this article.

[28] For numerical convenience we have chosen the site energies $\epsilon$ and hopping amplitudes $\beta$ in Eq. (11) to be the same for all of the ideal one-dimensional tight-binding leads that represent the electrodes, and to be independent of the applied bias voltage. Hence in Eq.(12) $T=\sum_{i=1}^{m} \frac{v^{\prime}}{v}\left|t_{i}\right|^{2}=\sum_{i=1}^{m}\left|\psi_{1, i}\right|^{2}$ since the velocities of the incoming electron $(v)$ and the (elastically scattered) transmitted electron $\left(v^{\prime}\right)$ are equal. However, the transmission probability $T$ depends on the applied bias voltage $V_{\text {bias }}$ due to the dependence of the molecular orbital 
energies on the bias that is calculated as described in Sec. IID.

[29] E. Emberly and G. Kirczenow, Phys. Rev. Lett. 81, 5205 (1998); J. Phys.: Condens. Matter 11, 6911 (1999).

[30] R. Landauer, IBM J. Res. Dev. 1, 223 (1957); Phys. Lett. 85A, 91 (1981).

[31] L. E. Ballentine, Quantum Mechanics: A Modern Development (World Scientific, Singapore, 1998), Chap. 19.

[32] The implementation used was that of G. Calzaferri, R. Rytz, and M. Brändle, ICON-EDiT, http://iacrs1.unibe.ch/program/iconedit.html

[33] W. Song and M. Yoshitake, Applied Surface Science 251, 14 (2005).

[34] E. G. Emberly and G. Kirczenow, Phys. Rev. B 64, 125318 (2001).

[35] The B3pW91 functional and Lanl2DZ basis set were used for this calculation. M. J. Frisch et al., GAUSSIAN 98, Gaussian, Inc., Pittsburgh, PA, 1998.

[36] In order to simulate strong molecule-substrate coupling on a uniform oxide surface, each substrate contact in this case is positioned 4.0 Ảbelow the plane of the molecule $(2.5 \AA$ from the nearest atom of the molecule). The tip probe is positioned 4.1 Aabove the molecule. See Fig, 2

[37] Changing the ratio $\alpha: \gamma_{1}: \gamma_{2}$ affects the energies of the relevant molecular orbitals when a bias voltage is applied, but does not qualitatively change the model results as long as the condition $\gamma_{2}<\alpha<\gamma_{1}$ is met. For a ratio $\alpha: \gamma_{1}: \gamma_{2}$ set to 9:10:8 instead of 3:4:2 (corresponding to smaller differences in the electrostatic effects on each orbital), all of the qualitative features described in Sec.III remain the same.

[38] Here, the substrate contact $S_{4}$ is positioned 4.0 Abelow the plane of the molecule, with the other three substrate contacts positioned 4.5 Åbelow the plane. The tip probe is positioned 4.5 Åabove the molecule.

[39] Since the LUMO2 is strongly coupled to the substrate (similar to case A: Strong fourfoldsymmetric molecule-substrate coupling), we assume the splitting of degeneracy primarily changes the LUMO1 energy: $\epsilon_{L U M O 1}=\epsilon_{L U M O}+0.5 \mathrm{eV}, \epsilon_{L U M O 2}=\epsilon_{L U M O}$.

[40] Here, the substrate contacts are positioned 4.5 Ảbelow the plane of the molecule (3.0 Afrom the nearest atom of the molecule) and the tip probe is positioned 4.0 Aabove the molecule. The lateral electrode positions are the same as for the other cases (see Fig,2).

[41] S. W. Wu, N. Ogawa, and W. Ho, Science 312, 1362 (2006). 
FIG. 1: (Color Online) A schematic energy level diagram of a transition from an occupied electron state (incoming from the tip probe on the left, shown in red) to an unoccupied electron state (incoming from a substrate contact on the right, shown in blue). A photon is created with energy $\hbar \omega$, equal to the difference in energy between the two electron states. The dashed lines represent the molecular portions of the states.

FIG. 2: (Color Online) The Zn(II)-etioporphyrin I molecule, showing substrate contacts $\mathrm{S}_{1}, \mathrm{~S}_{2}, \mathrm{~S}_{3}$, and $\mathrm{S}_{4}$ (open blue circles, into the page) and the tip probe (blue dot, out of page). Carbon atoms are red, nitrogen atoms are green, the zinc atom is yellow, and hydrogen atoms are white.

FIG. 3: (Color Online) Strong fourfold-symmetric coupling between molecule and substrate: Electric current, and molecular orbital energies as a function of bias voltage. (a) Approach A, I vs. $V_{\text {bias. }}$. Red lines represent dI/dV. (b) Approach B, I vs. $V_{\text {bias }}$. (c) Approach A, molecular orbital energies (dashed lines represent tip and substrate electrochemical potentials). (d) Approach B, molecular orbital energies.

FIG. 4: (Color Online) From Qiu et al.[17]. Reprinted with permission from AAAS. Experimental $\mathrm{dI} / \mathrm{dV}$ curves for Zn-etioporphyrin $/ \mathrm{Al}_{2} \mathrm{O}_{3} / \mathrm{NiAl}(110)$ obtained with the STM, for molecules at different locations on the substrate. (A-F) dI/dV curves representative of the various molecular images observed. The curve seen in B was most commonly observed (30\% of the time). Molecular electroluminescence was observed for cases A and B but not for C-F.

FIG. 5: (Color Online) Localized strong coupling: photon emission, electric current, and molecular orbital energies as a function of bias voltage. (a) Approach A, photon emission vs. $V_{\text {bias }}$. (b) Approach B, photon emission vs. $V_{\text {bias }}$. (c) Approach A, I vs. $V_{\text {bias }}$. Red lines represent dI/dV. (d) Approach B, I vs. $V_{\text {bias }}$. Dashed line represents $E_{F}=-10.3 \mathrm{eV}$. (e) Approach A, molecular orbital energies. (f) Approach B, molecular orbital energies.

FIG. 6: Localized strong coupling: Onset of photon emission, at the HOMO-LUMO2 emission peak. (a) Approach A, emission rate vs. photon energy, for three different values of $V_{\text {bias }}$ around the onset voltage. (b) Approach B, emission rate vs. photon energy. 
FIG. 7: (Color Online) From Qiu et al.[17]. Reprinted with permission from AAAS. Experimental photon emission spectra for molecules corresponding to Fig, $4 \mathrm{~B}$, for various bias voltages around the onset voltage.

FIG. 8: Weak fourfold-symmetric coupling: photon emission, electric current, and molecular orbital energies as a function of bias voltage. (a) Approach A, photon emission vs. $V_{\text {bias }}$. (b) Approach B, photon emission vs. $V_{\text {bias }}$. (c) Approach A, I vs. $V_{\text {bias }}$. (d) Approach B, I vs. $V_{\text {bias }}$. (e) Approach A, molecular orbital energies. (f) Approach B, molecular orbital energies. 
Initial electron state (occupied)

- Final electron state (unoccupied)

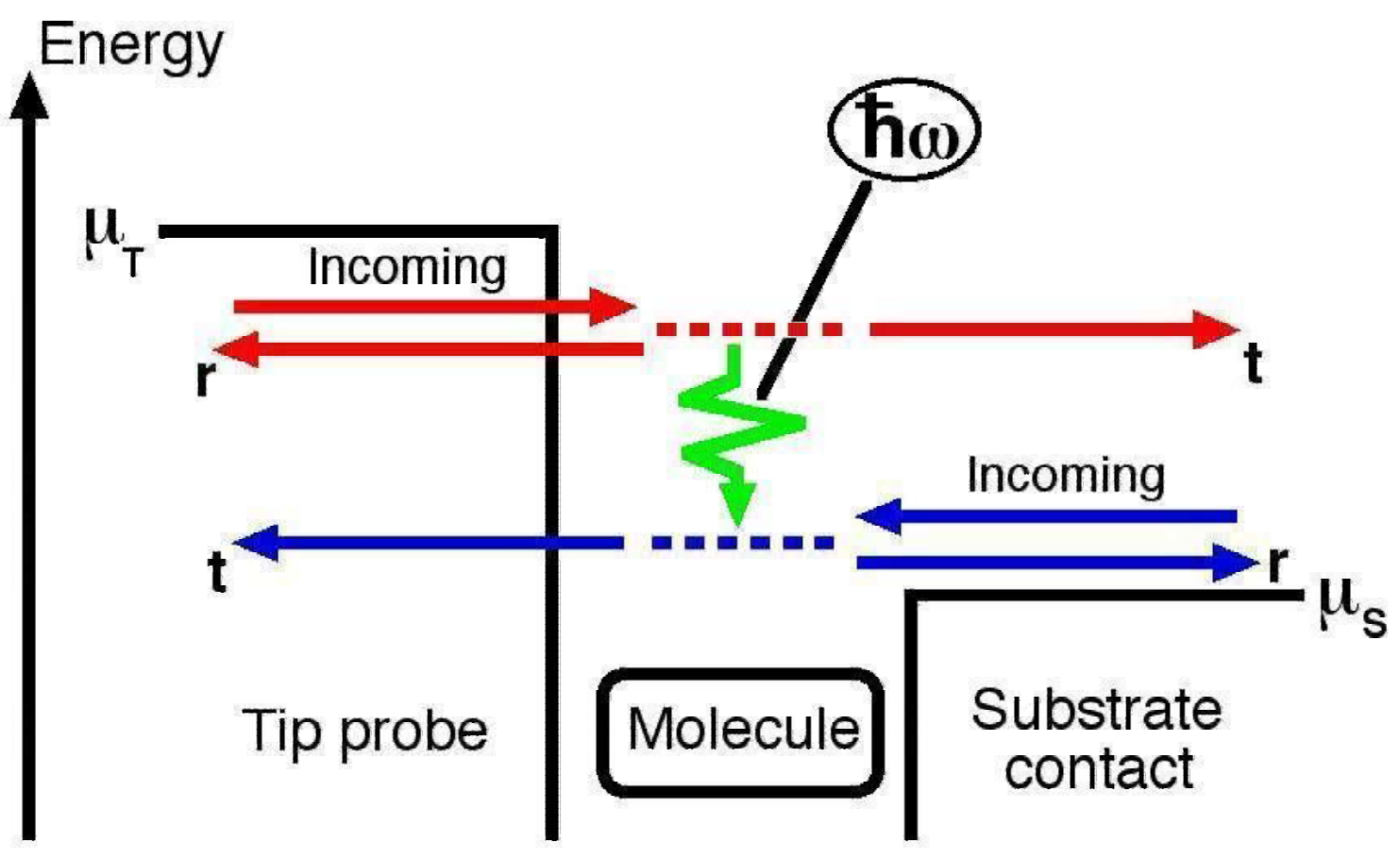

Buker and Kirczenow, Fig. 1 


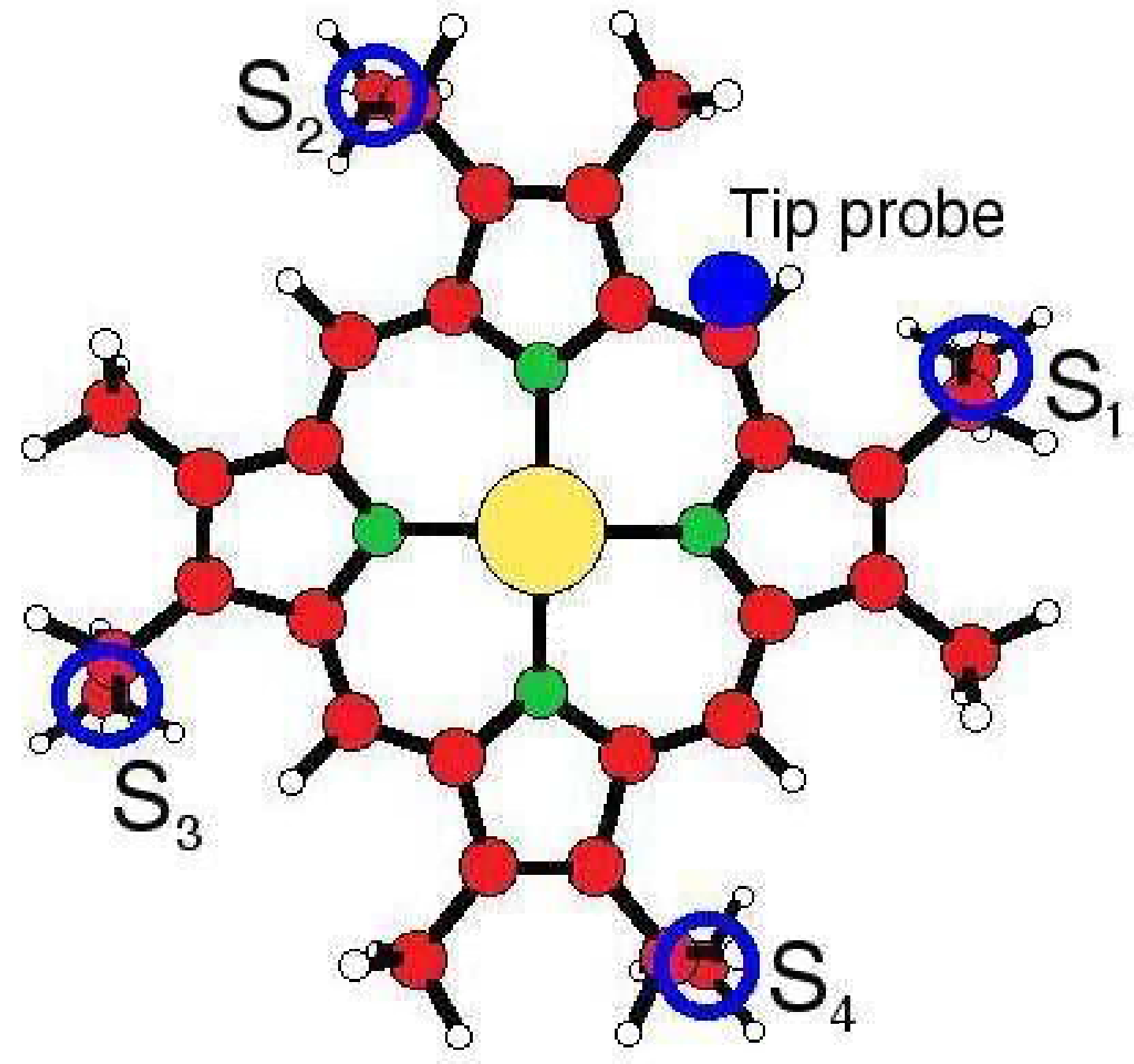

Buker and Kirczenow, Fig. 2 

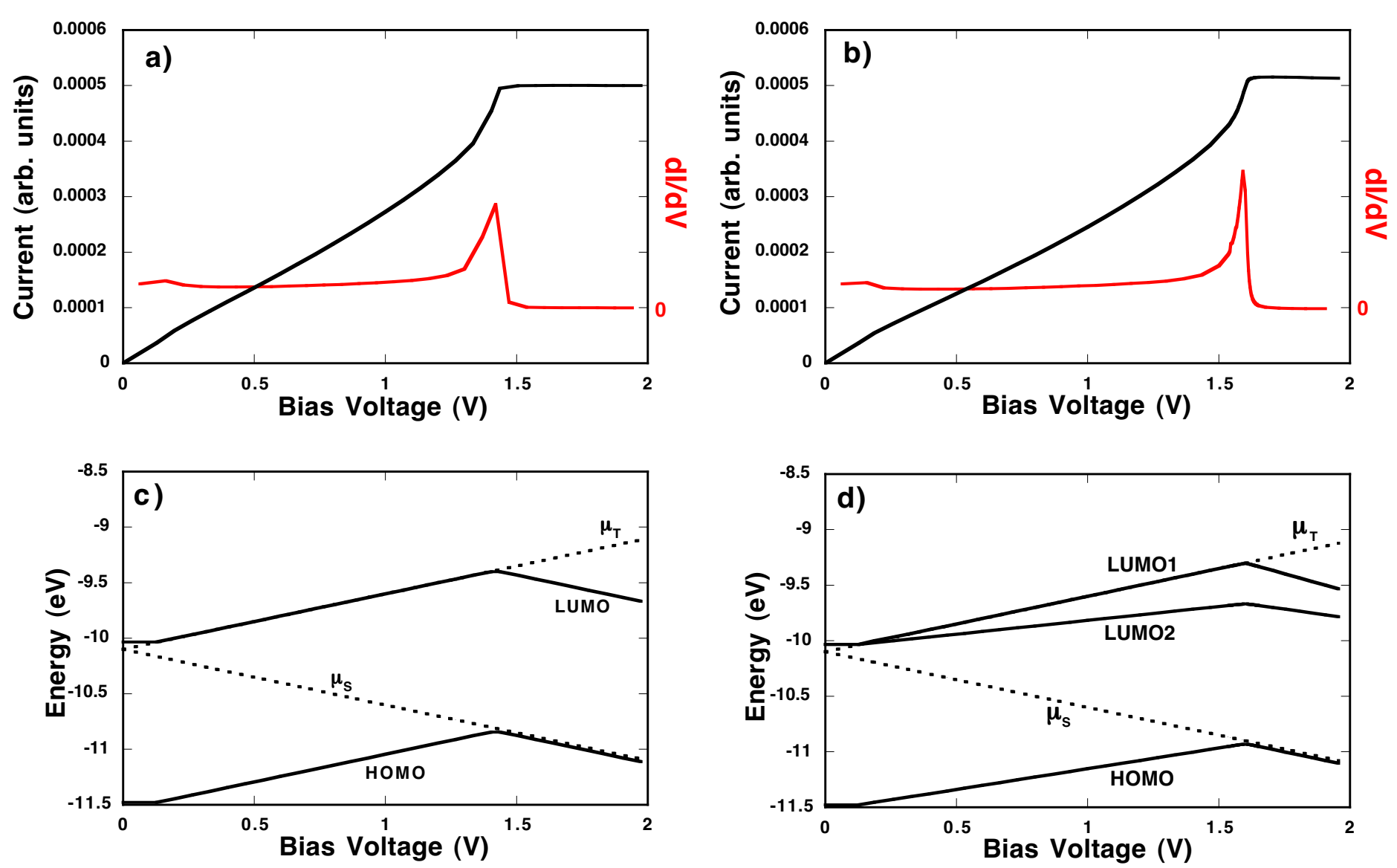

Buker and Kirczenow, Fig. 3 


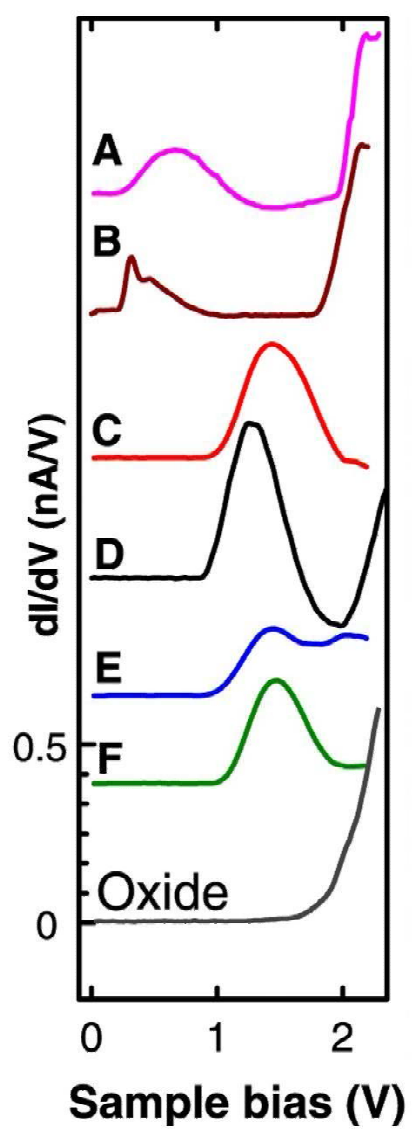

Buker and Kirczenow, Fig. 4 

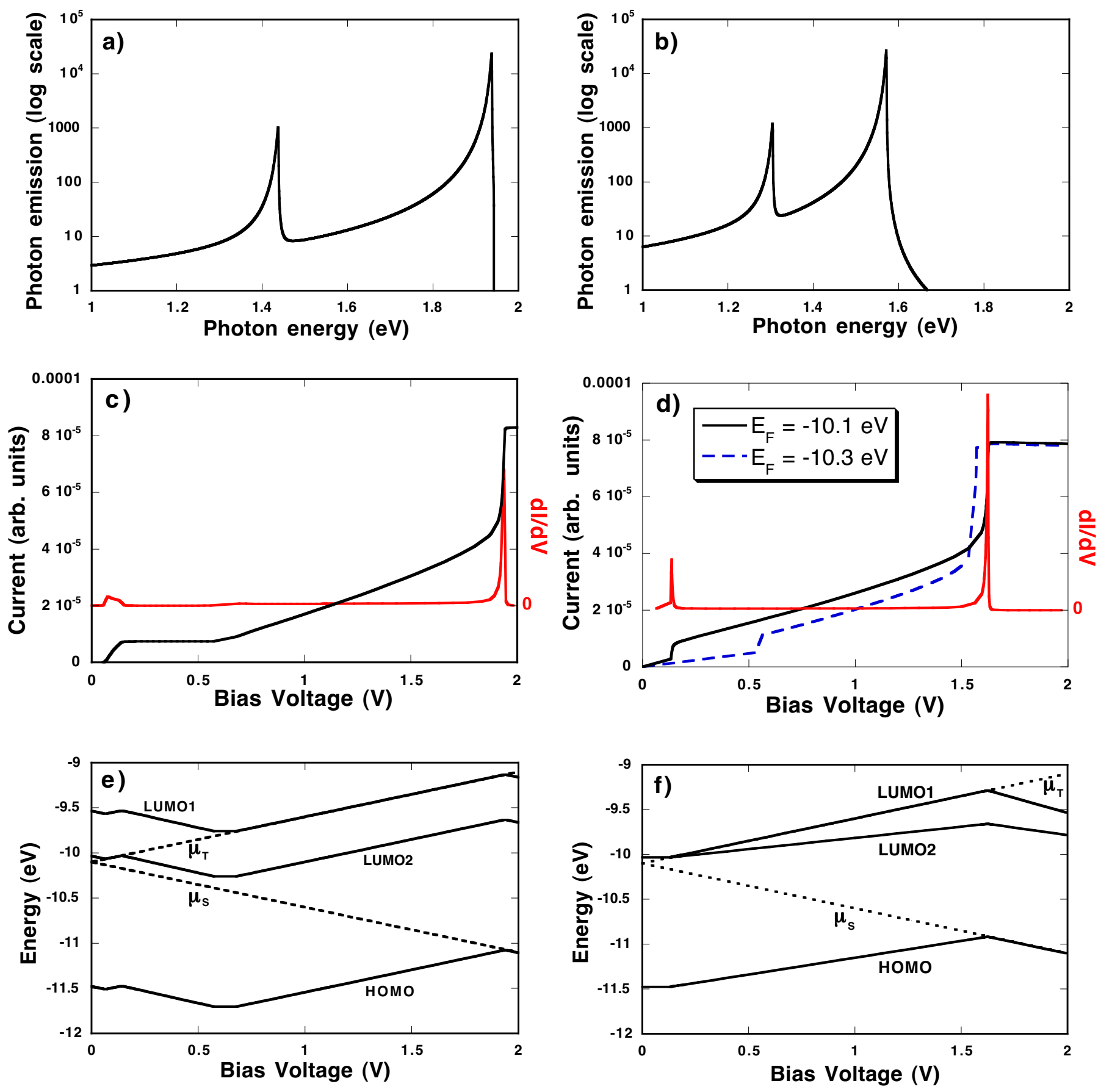

Buker and Kirczenow, Fig. 5 

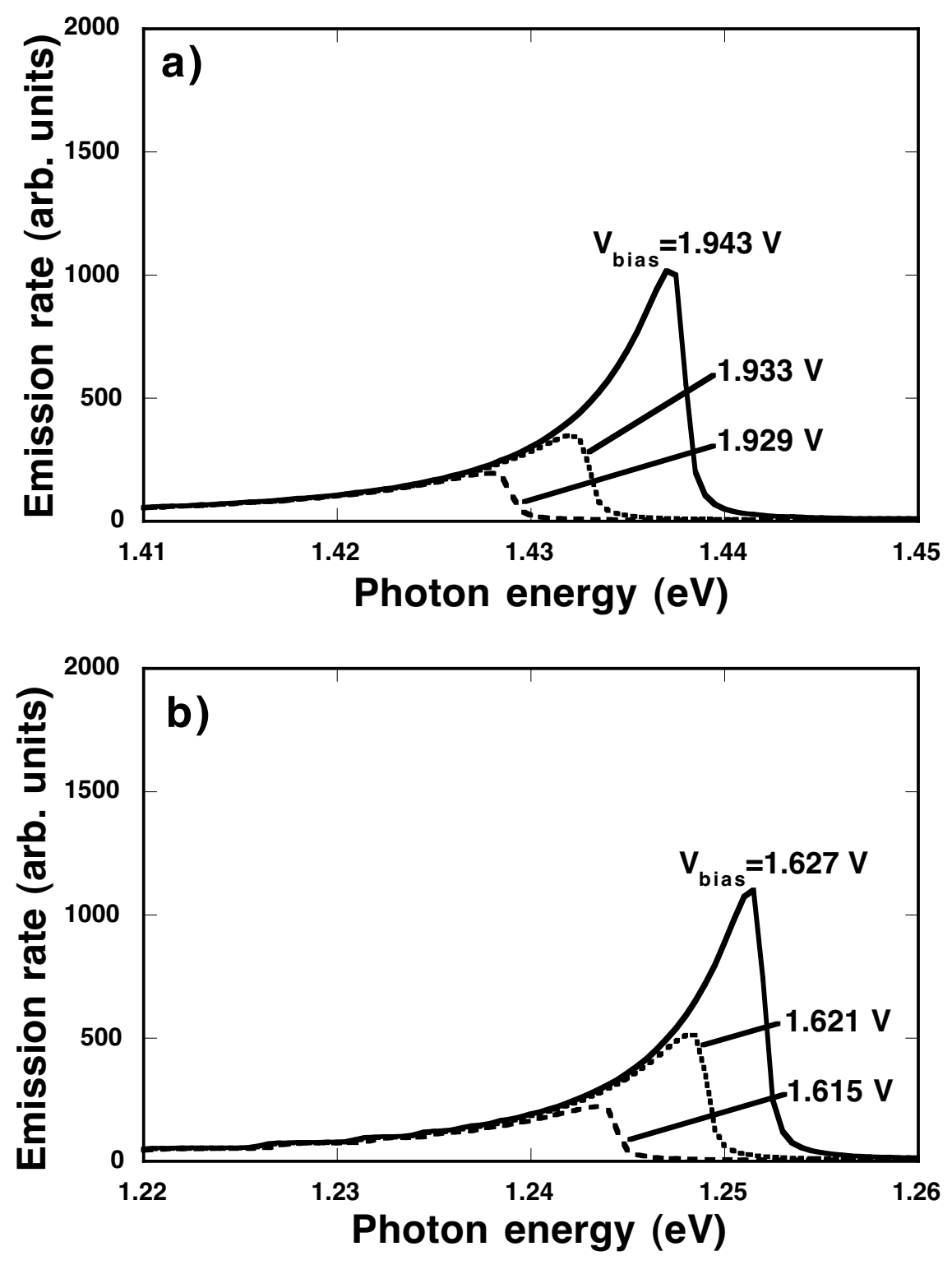

Buker and Kirczenow, Fig. 6 


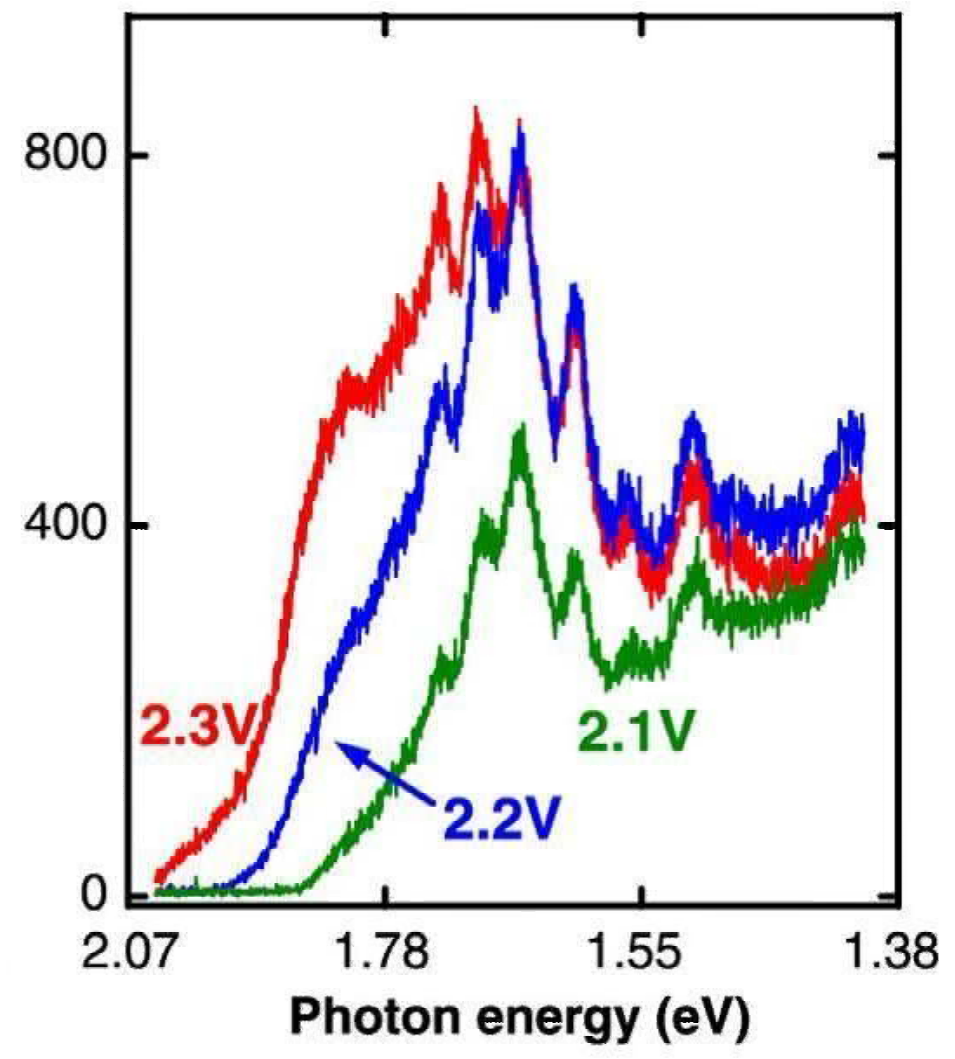

Buker and Kirczenow, Fig. 7 

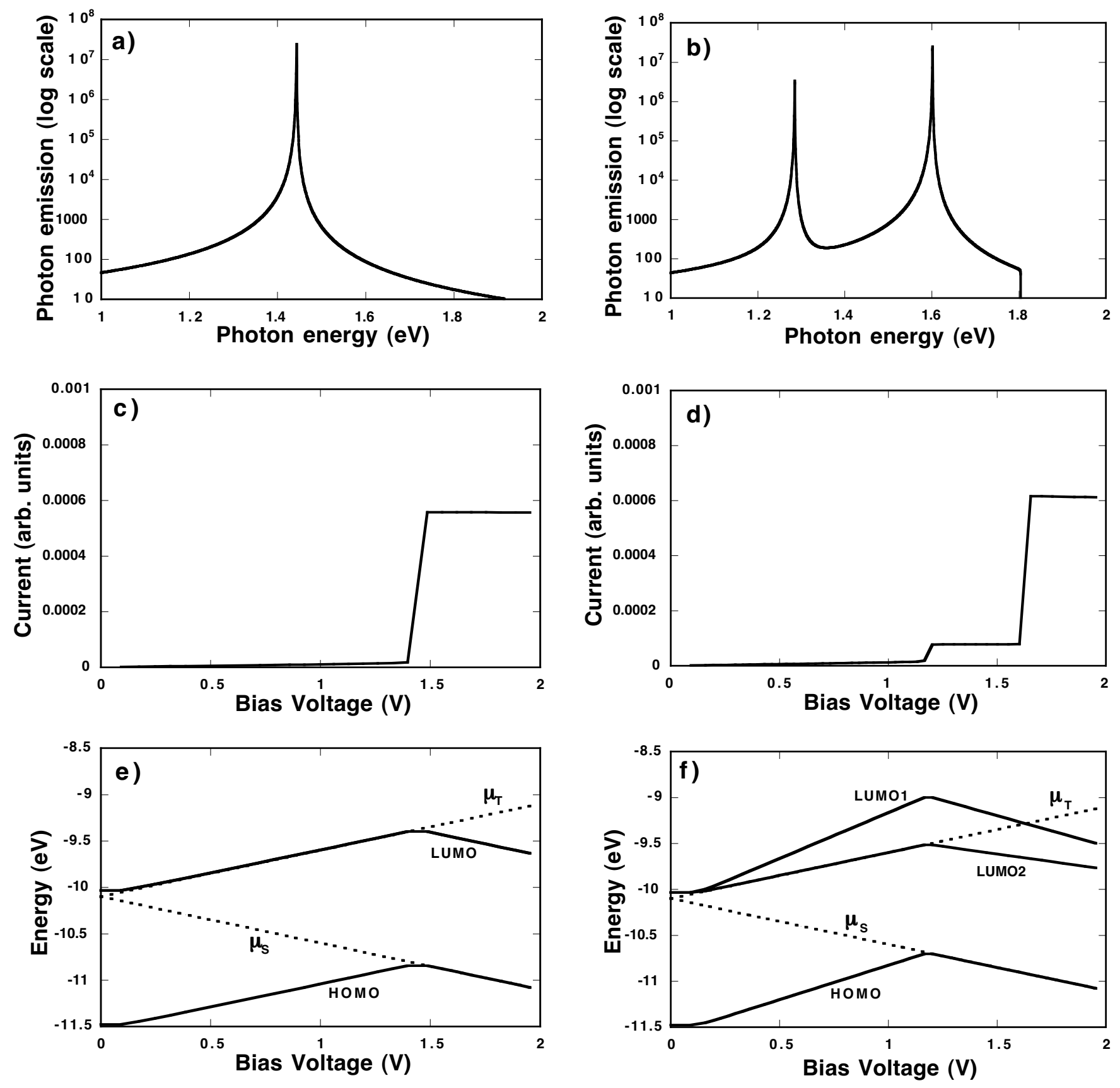

Buker and Kirczenow, Fig. 8 